\title{
A CONTRIBUIÇÃO DAS UNIVERSIDADES PARA O DESENVOLVIMENTO DA AGRICULTURA NO BRASIL ${ }^{1}$
}

\author{
Erly Cardoso Teixeira ${ }^{2}$ \\ Felippe Clemente ${ }^{3}$ \\ Marcelo José Braga ${ }^{4}$
}

RESUMO: As principais contribuições das universidades para o desenvolvimento do agronegócio brasileiro são a formação de capital humano e a geração de tecnologias. Os profissionais de alta qualidade formados nas universidades foram alocados para a pesquisa, o ensino, a extensão e para o desempenho de cargos executivos nos diversos elos das cadeias do agronegócio. As pesquisas realizadas nas universidades, via teses de doutorado e mestrado, em número de 7824, apenas no triênio 2007-09, promoveram mudanças tecnológicas de grande relevância para o desenvolvimento do agronegócio.

Palavras-chave: Formação de capital humano, pesquisa, mudança tecnológica.

\begin{abstract}
The universities main contributions to the development of Brazilian agribusiness are human capital formation and technology generation. High-quality professionals trained in universities are allocated to research, teaching, extension and as managers in the various links of the agribusiness chains. The research carried out by universities via doctoral dissertations and masters promoted technological changes of great significance for the development of agribusiness. More than 7800 theses from the graduate programs in agrarian science were defended in the period of 2007-09.
\end{abstract}

Keywords: Human capital formation, research, technological change.

1 Artigo escrito para apresentação no XXV Seminário Internacional de Política Econômica, 09 a 10/10/2013, Viçosa, MG. Os autores são agradecidos à Fapemig pelo apoio financeiro.

2 Ph.D., Professor Títular, Universidade Federal de Viçosa, Viçosa, Minas Gerais, Brasil. E-mail: teixeira@ufv. $\underline{\text { br }}$

3 Doutorando em economia aplicada, Universidade Federal de Viçosa. E-mail: felippe.clemente@ufv.br

4 D.Sc., Professor Associado, Universidade Federal de Viçosa. E-mail: mjbraga@ufv.br 


\section{Introdução}

O agronegócio brasileiro, com PIB de R \$ 928,8,0 bilhões, gerou 22,5 \% da renda nacional em 2012 e cresceu 5,7\% em 2011 e -1,4\% em 2012. A balança comercial do agronegócio apresentou saldo de US $\$ 76,0$ bilhões em 2011 e de US\$ 79,0 bilhões em 2012 (Cepea). Além disso, o país é o maior exportador de açúcar, café, suco de laranja, álcool e carne de frango; o segundo maior exportador de soja; e o terceiro, de carne bovina (Mapa). A produtividade total dos fatores (PTF) cresceu 119\% entre 1970 e 2006, resultando numa taxa de crescimento médio para o período de 2,2 \% ao ano (MENDES et al., 2010). Considerando o período de 1975 a 2011, Gasques et al. (2012) encontraram uma taxa média de crescimento da PTF de 3,56\% ao ano. Esse crescimento do agronegócio brasileiro está fortemente ligado às pesquisas realizadas nas universidades e nos centros de pesquisa, aos serviços de extensão, às políticas agrícolas e ao empreendedorismo dos empresários rurais brasileiros.

Barret, Cartes e Timmer (2010) destacam as contribuições de Theodore Schultz, que evidencia o crescimento da produtividade agrícola por meio da mudança técnica. Em seu trabalho "Transforming traditional agriculture”, Schultz (1964) desenvolve um quadro para explicar a hipótese dos produtores "pobres, mas eficientes". O autor argumenta que a chave para promover o crescimento é fornecer aos agricultores novas formas de rentabilidade alicerçadas no conhecimento e nos preços competitivos dos insumos. Schultz (1971) também destaca o papel da educação para os trabalhadores rurais, a fim de lidar com situações de desequilíbrio causadas pelas novas tecnologias. Schultz (1964) enfatiza dois importantes elementos para promover o desenvolvimento da agricultura: capital humano e desenvolvimento tecnológico. Essas ideias têm influenciado a academia e os formuladores de políticas no Brasil.

A pesquisa agrícola foi feita inicialmente nas escolas agrícolas, criadas no final do século XIX. De acordo com Sediyama et al. (2012), a criação das escolas agrícolas criou um privilegiado local para a construção científica e técnica do conhecimento, os quais foram acumulados e aplicados na 
produção agrícola. Posteriormente, esse esforço foi ampliado com a criação dos institutos de pesquisa e dos centros estaduais e federais de pesquisa. Nesse trabalho, tratar-se-á apenas da contribuição das universidades para o desenvolvimento do agronegócio.

O artigo está organizado em três seções, além dessa introdução. O próximo capítulo descreve a contribuição das universidades na formação de capital humano. A parte seguinte traz as contribuições das universidades na inovação técnica via pesquisa e desenvolvimento tecnológico. Finalmente, a última seção apresenta as conclusões do trabalho.

\section{Contribuição das universidades para a formação de capital humano}

\subsection{Formação de Profissionais de alta qualidade técnica}

Uma importante contribuição das universidades para o desenvolvimento do agronegócio brasileiro foi suprir com profissionais de boa qualidade técnica os centros de pesquisa, os serviços de extensão, os diversos elos da cadeia do agronegócio e o sistema de ensino das ciências agrárias. Mas como foi possível a essas escolas e universidades formar esses profissionais de alta qualidade técnica? A resposta a essa pergunta pode ser encontrada na fundação em bases sólidas dessas instituições de ensino, na criação da Capes e CNPq em 1951 e nos convênios firmados entre universidades brasileiras e americanas no final da década de 1950.

\subsection{Fundação em bases sólidas}

No século XIX, conforme Sediyama et al. (2012), as primeiras escolas agrícolas brasileiras a conferir o título de agrônomo foram: a "Escola 
Superior de Agronomia Taquariense", no Rio Grande do Sul, em 1896; a "Escola Politécnica do Rio de Janeiro, em 1896; a "Escola Politécnica de São Paulo, em 1898; e a "Escola de Engenharia de Porto Alegre, em 1897. As universidades brasileiras dedicadas ao ensino das ciências agrárias foram criadas em bases sólidas, no início do século $\mathrm{XX}$, refletindo a experiência dos Land-Grant Colleges americanos e da tradição europeia de bom ensino das técnicas agrícolas. As instituições Escola Superior de Agricultura Luiz de Queiroz (ESALQ-USP), criada em 1901; a Escola Superior de Agricultura de Lavras, hoje Universidade Federal de Lavras (UFLA), em 1908; e a Escola Superior de Agricultura e Veterinária, hoje Universidade Federal de Viçosa (UFV), fundada em 1922, sempre buscaram manter um corpo docente de alta qualidade técnica apoiado na pesquisa, na experimentação, no ensino e na extensão dos conhecimentos.

\subsection{Criação da Capes e do CNPq}

O aprimoramento do corpo docente das universidades nos graus de mestre e doutor tornou-se mais rápido a partir da criação da Coordenação de Aperfeiçoamento de Pessoal do Ensino Superior (Capes) e do Conselho Nacional de Desenvolvimento Científico e Tecnológico (CNPq) em 1951. Essas duas instituições concederam bolsas de estudo para treinamento no país e no exterior, fortalecendo os programas de graduação das universidades e os programas de pós-graduação criados apenas a partir de 1961. A Tabela 1 mostra o número de bolsas concedidas pela Capes nos primeiros anos de sua instalação.

Tabela 1. Número de bolsas de estudos concedidas - Capes

\begin{tabular}{lccc}
\hline Bolsas de Estudos & 1953 & 1954 & Variação \\
\hline Graduação no Brasil & 2 & 32 & $1.500 \%$ \\
Pós-Graduação no Brasil & 23 & 51 & $122 \%$ \\
Graduação no Exterior & 54 & 72 & $33 \%$ \\
\hline
\end{tabular}

Fonte: Capes. 
Atualmente, conforme Tabela 2, o país conta com mais de 5.000 cursos de mestrado (M.Sc.), doutorado (D.Sc.) e mestrado profissional (M.Sc.P). $\mathrm{Na}$ área das Ciências Agrárias, são 588 cursos de pós-graduação, tendo sido concedidas mais de 7.000 bolsas de mestrado e doutorado pelo CNPq entre 1996 e 2008 (Tabela 3). Nos programas de pós-graduação das Ciências Agrárias, entre 2007-2009, foram produzidas 7.824 teses (Capes). Essas pesquisas evidenciam a grande contribuição das universidades para o desenvolvimento da agricultura no Brasil. No período de 2010 a 2012, espera-se que tenham sido defendidas mais de 8.000 teses de mestrado e doutorado nas ciências agrárias. Portanto, apenas no período recente dos últimos seis anos, foram feitas mais de 15.000 pesquisas com o objetivo de resolver problemas tecnológicos do agronegócio brasileiro. As universidades continuam a fornecer mestres e doutores para os centros de pesquisa, para a extensão rural e para as cadeias do agronegócio. Esses egressos levam com eles o que as universidades têm de mais avançado em termos de estrutura laboratorial, métodos e tecnologia de pesquisa.

Tabela 2. Programas de Pós-Graduação no Brasil, 2012

\begin{tabular}{ccccc}
\hline Grande Área & M.S. & D.S. & M.S.P. & Total \\
\hline Ciências Agrárias & 349 & 218 & 21 & $\mathbf{5 8 8}$ \\
Ciências Biológicas & 259 & 191 & 17 & $\mathbf{4 6 7}$ \\
Saúde & 455 & 332 & 86 & $\mathbf{8 7 3}$ \\
Ciências Naturais & 275 & 174 & 15 & $\mathbf{4 6 4}$ \\
Ciências Humanas & 452 & 251 & 23 & $\mathbf{7 2 6}$ \\
Ciências Sociais Aplicadas & 346 & 162 & 81 & $\mathbf{5 8 9}$ \\
Engenharias & 306 & 163 & 64 & $\mathbf{5 3 3}$ \\
Línguas, Literatura e Artes & 177 & 99 & 4 & $\mathbf{2 8 0}$ \\
Multidisciplinar & 309 & 152 & 145 & $\mathbf{6 0 6}$ \\
\hline Total & $\mathbf{2 9 2 8}$ & $\mathbf{1 7 4 2}$ & $\mathbf{4 5 6}$ & $\mathbf{5 1 2 6}$ \\
\hline
\end{tabular}

Fonte: Capes. 
Tabela 3 - Número de bolsas de estudos concedidas pelo CNPq para a Grande Área Ciências Agrárias, de 1996 a 2003 para programas de mestrado, e de 1996 a 2008 para programas de doutorado

\begin{tabular}{ccc}
\hline Ano & M.S. & D.S. \\
1996 & 757 & 152 \\
1997 & 629 & 147 \\
1998 & 546 & 181 \\
1999 & 382 & 171 \\
2000 & 485 & 176 \\
2001 & 443 & 214 \\
2002 & 525 & 208 \\
2003 & 532 & 256 \\
2004 & & 243 \\
2005 & & 290 \\
2006 & & 361 \\
2007 & & 378 \\
2008 & & 412 \\
\hline Total & $\mathbf{4 , 2 9 9}$ & $\mathbf{3 , 1 8 9}$ \\
\hline
\end{tabular}

Fonte: CNPq.

2.4. Implementação de convênios entre universidades brasileiras e americanas

A Capes e o CNPq foram fundamentais para a negociação e o financiamento de diversos programas entre universidades americanas e brasileiras, favorecendo a vinda de professores que ajudaram na criação dos primeiros programas de pós-graduação no Brasil em 1961. A Tabela 4 informa sobre os primeiros graus de mestre concedidos pelas universidades brasileiras. 
Tabela 4. Grau de Mestre concedido por universidades brasileiras

\begin{tabular}{ccccccccccc}
\hline Ano & UFV & ITA & COPPE & PUC/RJ & CBPF & IMPA & IF/USP & UFRJ & EP/ & Total \\
\hline 1961 & $\mathbf{5}$ & $\mathbf{0}$ & $\mathbf{0}$ & $\mathbf{0}$ & $\mathbf{0}$ & $\mathbf{0}$ & $\mathbf{0}$ & $\mathbf{0}$ & $\mathbf{0}$ & $\mathbf{5}$ \\
1962 & $\mathbf{8}$ & $\mathbf{0}$ & $\mathbf{0}$ & $\mathbf{0}$ & $\mathbf{0}$ & $\mathbf{0}$ & $\mathbf{0}$ & $\mathbf{0}$ & $\mathbf{0}$ & $\mathbf{8}$ \\
1963 & $\mathbf{9}$ & $\mathbf{4}$ & $\mathbf{0}$ & $\mathbf{0}$ & $\mathbf{0}$ & $\mathbf{0}$ & $\mathbf{0}$ & $\mathbf{0}$ & $\mathbf{0}$ & $\mathbf{1 3}$ \\
1964 & $\mathbf{1 8}$ & $\mathbf{3}$ & $\mathbf{8}$ & $\mathbf{1}$ & $\mathbf{0}$ & $\mathbf{0}$ & $\mathbf{0}$ & $\mathbf{0}$ & $\mathbf{0}$ & $\mathbf{3 0}$ \\
1965 & $\mathbf{1 6}$ & $\mathbf{9}$ & $\mathbf{8}$ & $\mathbf{0}$ & $\mathbf{6}$ & $\mathbf{2}$ & $\mathbf{0}$ & $\mathbf{0}$ & $\mathbf{0}$ & $\mathbf{4 1}$ \\
1966 & $\mathbf{2 3}$ & $\mathbf{9}$ & $\mathbf{6}$ & $\mathbf{6}$ & $\mathbf{2}$ & $\mathbf{0}$ & $\mathbf{0}$ & $\mathbf{0}$ & $\mathbf{0}$ & $\mathbf{4 6}$ \\
1967 & $\mathbf{3 0}$ & $\mathbf{1 0}$ & $\mathbf{2 2}$ & $\mathbf{2 0}$ & $\mathbf{1}$ & $\mathbf{2}$ & $\mathbf{2}$ & $\mathbf{0}$ & $\mathbf{0}$ & $\mathbf{8 7}$ \\
1968 & $\mathbf{2 3}$ & $\mathbf{1 1}$ & $\mathbf{3 8}$ & $\mathbf{1 6}$ & $\mathbf{1}$ & $\mathbf{5}$ & $\mathbf{5}$ & $\mathbf{1}$ & $\mathbf{0}$ & $\mathbf{1 0 0}$ \\
1969 & $\mathbf{4 5}$ & $\mathbf{1 3}$ & $\mathbf{2 3}$ & $\mathbf{2 6}$ & $\mathbf{0}$ & $\mathbf{1 5}$ & $\mathbf{1 3}$ & $\mathbf{0}$ & $\mathbf{4}$ & $\mathbf{1 3 9}$ \\
1970 & $\mathbf{3 9}$ & $\mathbf{1 9}$ & $\mathbf{5 2}$ & $\mathbf{3 5}$ & $\mathbf{4}$ & $\mathbf{4}$ & $\mathbf{1 9}$ & $\mathbf{2}$ & $\mathbf{2 9}$ & $\mathbf{2 0 3}$ \\
\hline Total & $\mathbf{2 1 6}$ & $\mathbf{7 8}$ & $\mathbf{1 5 7}$ & $\mathbf{1 0 4}$ & $\mathbf{1 4}$ & $\mathbf{2 8}$ & $\mathbf{3 9}$ & $\mathbf{3}$ & $\mathbf{3 3}$ & $\mathbf{6 7 2}$ \\
\hline
\end{tabular}

Fonte: Ferrari (2001).

Criados os programas de pós-graduação, esses professores, majoritariamente americanos, colaboravam, ministrando aulas, pesquisando e publicando, auxiliando na montagem dos laboratórios e na seleção dos alunos para fazerem o doutorado nas melhores universidades americanas. A presença desses professores teve grande influência no ensino da graduação e da pós-graduação, que adotou o rígido controle americano do aprendizado via disciplinas, trabalhos para casa, exames, avaliações e teses de mestrado e doutorado. Novamente, o papel da Capes e CNPq, por meio dos programas de cooperação entre universidades estrangeiras, foi muito relevante, concedendo bolsas de estudo para os professores que iam fazer o doutorado e bolsas de pesquisa para os professores que retornavam do doutorado.

O principal acordo entre as universidades americanas e brasileiras foi o da Purdue University com a UFV. O acordo entre a Purdue University e a UFV, assinado em 1959, foi financiado pela Capes, CNPq, Fundação Ford e Fundação Kellogs (Fernandez, 1991). Por esse acordo, estiveram na UFV cerca de 200 pesquisadores, técnicos e professores da Purdue University, no período de 1959 a 1970. A partir dessa cooperação, 
intensificou-se o treinamento de estudantes brasileiros no exterior. Noventa professores da UFV obtiveram títulos de mestrado e doutorado na Purdue University entre os anos de 1973 e 1976 (Tabela 5).

Tabela 5 - Professores da UFV treinados na Purdue University entre 1973 e 1976

\begin{tabular}{lccc}
\hline Ano & M.S. & D.S. & Total \\
\hline 1973 & 26 & 24 & 50 \\
1976 & 7 & 33 & 40 \\
\hline Total & 33 & 57 & 90 \\
\hline
\end{tabular}

Fonte: Fernandez (1991).

Os programas de pós-graduação da UFV contribuíram enormemente para a formação de pesquisadores qualificados para o sistema nacional de pesquisa e de extensão e para o gerenciamento das cadeias do agronegócio. Esses programas foram fundamentais para a realização de importantes trabalhos de pesquisa em melhoramento de plantas e animais, em solos e fertilidade, em mecanização, em manejo de plantas e animais e em criação de produtos que permitiram o desenvolvimento do agronegócio brasileiro. As pesquisas nas universidades prosseguem buscando respostas para problemas modernos, tais como mudança climática, controle de novas pragas e doenças, preservação ambiental. Técnicas modernas de engenharia genética, marcadores moleculares, identificação de genomas e desenvolvimento de organismos e culturas geneticamente modificados são explorados nos laboratórios.

\section{Contribuição das Universidades para a Pesquisa e Desenvolvimento}

A contribuição das universidades para o desenvolvimento da agricultura está relacionada com as pesquisas desenvolvidas em diversas áreas. Ou seja, as universidades são de fundamental importância na geração de 
tecnologias que permitiram e permitem o crescimento da agricultura no Brasil. Os próximos itens evidenciam algumas das contribuições mais importantes das instituições de pesquisa para o agronegócio brasileiro.

\subsection{Fertilidade do Solo}

Uma importante pesquisa gerada nas universidades foi a que permitiu a expansão da agricultura nas áreas de cerrado. As pesquisas que determinaram as dosagens de corretivos e de fosfatos para os solos do cerrado transformaram os milhões de hectares do deserto ou savana brasileira, como era tido até finais da década de 1960, num oásis de 79 milhões de hectares já na década de 1970 (NOVAIS et al., 2007). O Cerrado é o segundo maior bioma brasileiro, com 207 milhões de hectares, correspondendo a $24 \%$ do território nacional. Desse total, 139 milhões de hectares são terras cultiváveis, sendo que 79 milhões de hectares são atualmente cultivados. Aárea de Cerrado cultivada é ocupada com 54 milhões de hectares de pastagens; 21,6 milhões de hectares com culturas; e 3,4 milhões de hectares com áreas reflorestadas (Embrapa).

O primeiro experimento de campo envolvendo e fertilização na região de solos do Cerrado foi feito no início de 1900. Essa pesquisa confirmou que o solo do Cerrado era muito produtivo quando bem fertilizado (NOVAIS et al., 2007).

Em 1970, o Departamento de Ciência do Solo da North Carolina State University, EUA, inicia o Tropical Soils Research Project na América Latina em colaboração com pesquisadores de universidades e centros de pesquisa. Os objetivos do projeto eram rever, analisar e interpretar a literatura publicada e outras fontes de informação relacionadas com os fatores do solo que influenciavam a produção das culturas nas regiões tropicais na América Latina. Pesquisadores do recém-criado Centro de Pesquisas Agropecuárias do Cerrado (CPAC), em 1975, em estreita colaboração com os pesquisadores da Cornell University e da North Carolina State University, EUA, desenvolveram pesquisas sobre os 
mais diversos aspectos da fertilidade dos solos sob vegetação de Cerrado (NOVAIS et al., 2007). A Tabela 7 mostra a contribuição do Cerrado para a produção nacional em 2007.

Tabela 7. Contribuição do Cerrado na produção doméstica - 2007

\begin{tabular}{cc}
\hline Colheita & $\%$ \\
Algodão & 89.0 \\
Café & 48.0 \\
Cana-de-Açúcar & 10.0 \\
Carne & 55.0 \\
Feijão & 30.0 \\
Milho & 26.0 \\
Soja & 63.5 \\
Sorgo & 81.0 \\
\hline
\end{tabular}

Fonte: Faleiro e Sousa (2007).

\subsection{Milho Híbrido}

Um importante resultado da pesquisa realizada nas universidades surgiu já na década de 1930. Tratava-se do desenvolvimento das primeiras linhagens do milho híbrido brasileiro pelos pesquisadores Carlos Arnaldo Krugg, no Instituto Agronômico de Campinas (IAC), e Antônio Secundino de São José Araújo e Gladstone de Almeida Drummond, na UFV (Coelho, 1992). Rapidamente, o milho híbrido, divulgado pela extensão rural e comercializado pela empresa Agroceres $^{5}$, criada por Secundino e colaboradores em 1945, tornou-se a semente de milho mais plantada no País ${ }^{6}$. O Brasil foi o segundo país a desenvolver o milho híbrido e a adotá-lo nos plantios comerciais. Recentemente,

5 A Agroceres tornou-se a maior empresa produtora de sementes de milho híbrido e de hortaliças do país. Foi adquirida pela Monsanto em 1999.

6 A produção de milho em 2012 foi de 72,8 milhões de toneladas. 
as universidades continuam desenvolvendo hibridações de milho, como UFVM 100, UFVM 200, UFLA JM100, UENF 01 e UENF 14. Entretanto, os cultivares desenvolvidos nas universidades não representa mais que $2 \%$ do milho plantado no país. O principal fator para a redução da participação das universidades é que tem crescido a participação de companhias multinacionais no desenvolvimento de novos cultivares (SEDIYAMA et al., 2012).

\subsection{Café}

As elevadas taxas de retorno dos investimentos em pesquisa e extensão na cultura do café em Minas Gerais no período de 1970 a 1990 são apresentadas na tese de Ferreira (1993). As pesquisas com café foram desenvolvidas pelas Universidades Federais de Lavras e de Viçosa, pelo Instituto Brasileiro do Café (IBC) e pela Empresa de Pesquisa Agropecuária do Estado de Minas Gerais (Epamig), e a extensão foi feita pelo (IBC) e pela Empresa de Assistência Técnica e Extensão Rural do Estado de Minas Gerais (Emater-MG). A taxa interna de retorno obtida foi de $82 \%$ ou $108 \%$, dependendo se os benefícios tivessem sido iniciados em 1973 ou 1975, respectivamente. Essas pesquisas permitiram a expansão da produção brasileira de café de 20 milhões de sacas em 1960 para as atuais 50 milhões de sacas, e a produtividade passou de 10 sacas por hectare para mais de 20 sacas por hectare em Minas Gerais (CAFEICULTURA, 2007). Outro resultado da pesquisa e da extensão com café foi tornar o estado de Minas Gerais o maior produtor de café do país com mais de $50 \%$ da produção.

\subsection{Soja}

As pesquisas das universidades também contribuíram para o desenvolvimento de variedades de soja. A primeira experiência com soja no Brasil ocorre em 1882, na Bahia. Em São Paulo, as primeiras variedades de soja são introduzidas pelo IAC em 1889, sob coordenação 
de Franz W. Dafert (SEDIYAMA et al., 2012). Em Minas Gerais, o programa de melhoramento genético de soja da UFV teve início em 1963, por meio do convênio com a Purdue University (EUA), com os pesquisadores americanos Marvin L. Sweatingin e Kirk L. Athow, com o objetivo de desenvolver variedades adaptadas às áreas de Cerrado, localizadas na região Central do Brasil. Na época, foram trazidas para a UFV diversas variedades de soja da região Sul dos Estados Unidos. Entretanto, a sensibilidade da soja ao fotoperíodo fez com que a maioria dos materiais introduzidos apresentasse pequena estatura, sem condições de ser colhido mecanicamente, o que inviabilizava economicamente a cultura. Após uma série de ensaios, destacaram-se duas linhagens: a Mineira e a Viçoja, lançadas em 1969, pelo Professor Tuneo Sediyama. Essas variedades eram indicadas para solos naturalmente férteis ou de fertilidade corrigida. Desde essa época, mais de 2.000 tipos de hibridações foram feitas, sempre priorizando características como produtividade, adaptação à colheita mecanizada, boa qualidade fisiológica da semente, maturação uniforme, elevados teores de óleo e de proteína e resistência às doenças. Ressalta-se o intercâmbio constante entre a UFV e universidades e órgãos do exterior. Para as pesquisas de soja, fazem parte as Universidades de Purdue e Iowa (EUA), AVRDC (Taiwan) e Tsukuba (Japão). Nos anos 1980, a UFV incluiu em suas pesquisas o Programa de Qualidade de Soja para consumo humano, conduzindo testes para nível de óleo e proteína e eliminação de fatores antinutricionais. Em 2000, foram criados mais dois programa de pesquisas para a soja: um para a produção de biodiesel e outro para alimentação animal. Em 2011, a UFV contava com 58 cultivares criados na instituição, com 33 registrados e protegidos pelo Serviço Nacional de Proteção de Cultivares (SNPC) (SEDIYAMA et al., 2012). Os cultivares desenvolvidos na UFV têm contribuído para o aumento da produtividade e da qualidade da soja no Brasil, na Bolívia, no Paraguai e em diversos países da América Central. Atualmente, a produtividade média nacional da soja é $3.106 \mathrm{~kg} /$ ha. A pesquisa com soja gerou variedades que hoje são cultivadas desde as regiões temperadas do sul do país, $-33^{\circ}$ de latitude, até na região Norte, onde ultrapassa a linha do equador (SEDIYAMA et al., 2012). 


\subsection{Eucalipto}

A introdução do eucalipto no Brasil ocorre em 1868, sendo que, em 1903, o pesquisador Edmundo Navarro de Andrade, considerado o pioneiro do reflorestamento no país, inicia as pesquisas com eucalipto na Companhia Paulista de Estradas de Ferro. Na década de 1950, o eucalipto passa a ser plantado para fornecer matéria-prima para o abastecimento das fábricas de papel e celulose e, nos finais dos anos 1970 e início de 1980, as principais universidades e institutos de pesquisas brasileiros desenvolvem a clonagem, que ganha escala comercial. Com isso, a partir de 1990, o Brasil se torna referência mundial na eucaliptocultura. De acordo com Abraf (2007), as plantações de eucalipto ocupam mais de 3 milhões de hectares, respondendo por 3,5\% do PIB brasileiro. A exportação de produtos derivados de florestas plantadas somou US\$ 6,1 bilhões, dos quais $70 \%$ foram resultantes do cultivo do eucalipto. Com toda essa importância do setor, os institutos de pesquisas iniciaram trabalhos com o objetivo de desenvolver árvores mais adaptadas a diferentes solos e condições climáticas, agregando características de interesse econômico e social, como aumento de produtividade e maior capacidade de adaptação, resistência a algumas doenças e pragas e tolerância a condições de estresse. Essas variedades geneticamente melhoradas apresentam produtividade até dez vezes maior que em países como Finlândia, Portugal e Estados Unidos. Em algumas empresas florestais no país, que, na década de 1970, produziam uma média de $20 \mathrm{~m}^{3} / \mathrm{ha} / \mathrm{ano}$, hoje conseguem atingir até 50 $\mathrm{m}^{3} /$ ha/ano com o uso de material genético melhorado e das tecnologias disponíveis. Do eucalipto, foi obtida a celulose de fibra curta, cujas exportações hoje estão direcionadas a todo o mercado internacional para a produção de papel de imprimir e escrever. A celulose de eucalipto é alternativa para a celulose de fibra longa de pinus, árvore que na Europa só atinge o ponto de corte em 30 anos, período quatro vezes maior que o do eucalipto brasileiro. A Figura 1 mostra a diferença na produtividade dos principais países produtores de eucalipto. 


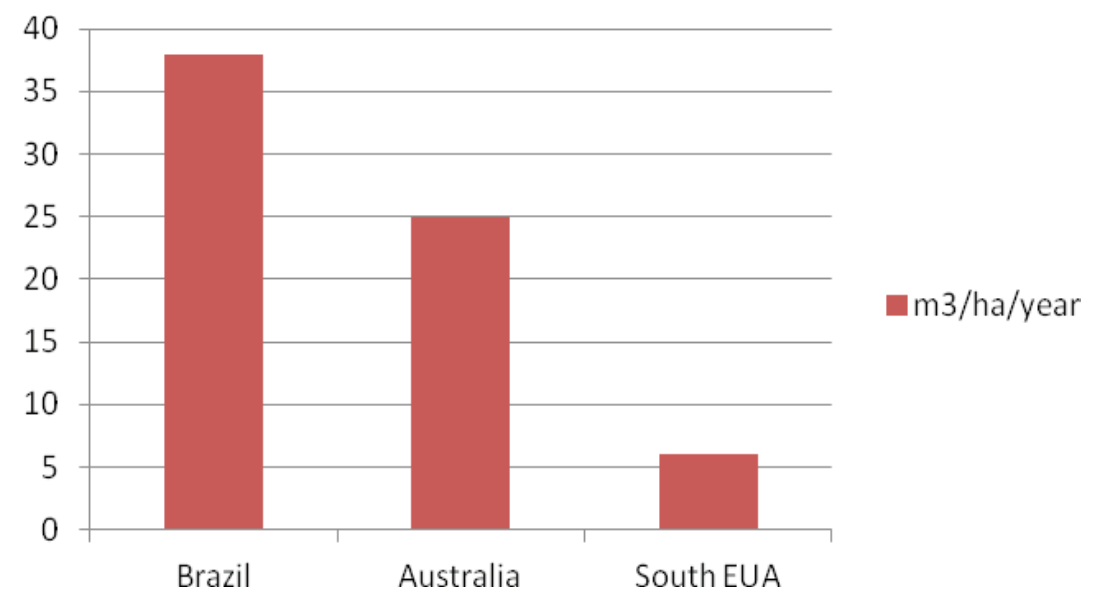

Figura 1 - Produtividade do Eucalipto no Brasil, Austrália e EUA Fonte: Abraf (2006).

As pesquisas atuais com eucalipto desenvolvidas nas universidades vão desde o sequenciamento genômico ao controle biológico de pragas e doenças, ao melhoramento genético utilizando marcadores moleculares para produção de características desejáveis até a produção de organismos geneticamente modificados.

\subsection{Cana-de-Açúcar}

As pesquisas voltadas para a cana-de-açúcar nas universidades ocorrem principalmente via a Rede Interuniversitária para o Desenvolvimento do Setor Sucro-energético - Ridesa, que envolve dez Universidades Federais. A rede foi formada em 1990 com as Universidades Federais do Alagoas (UFAL), de Pernambuco (UFRPE), de São Carlos (UFSCAR), do Rio de Janeiro (UFRJ) e Viçosa (UFV). Em 1992, passaram a fazer parte do programa a Universidade Federal do Paraná (UFPR) e a Universidade Federal de Sergipe (UFS); em 2003, a Universidade de Goiás (UFG); e em 2008, as Universidades do Mato Grosso (UFMT) e do Piauí (UFPI). A Ridesa envolve diversas parcerias público-privadas 
para o desenvolvimento de cultivares. Desde sua criação, a rede já desenvolveu 59 variedades de cana-de-açúcar, sendo responsável por 59\% da área cultivada no Brasil. Nos últimos cinco anos, 286 agrônomos foram treinados para trabalhar no setor, por meio do programa. Desde 2007, a Ridesa formou 35 mestres, 24 doutores e 7 pós-doutores, que desenvolvem pesquisas com cana-de-açúcar geneticamente modificada (SEDIYAMA et al., 2012).

\subsection{Avicultura}

Os trabalhos de genética e melhoramento de aves no Brasil foram iniciados na década de 1950. Por recomendação do Ministério da Agricultura, a ESALQ iniciou as pesquisas para melhoramento de posturas de aves e, posteriormente, direcionou trabalhos para aves de corte (COSTA, 1997 ). Em 1975, a UFV também ingressou nessa área de pesquisa. Essas duas instituições criaram convênios com empresas privadas para o desenvolvimento de pesquisas básicas, formação e treinamento de técnicos e obtenção de linhagens para produção de carne e ovos. Em 1979, o Instituto de Zootecnia de Nova Odessa - SP instalou um plano de obtenção de linhagens comerciais de poedeiras por meio da reconstituição de linhagens comerciais. Na década de 1980, a Universidade Federal de Santa Maria (UFSM), Rio Grande do Sul, passou a trabalhar no desenvolvimento de linhagens de postura de ovos vermelhos, com a finalidade de obter uma ave de dupla aptidão, ou seja, além de produtora de ovos, apresentaria peso corporal elevado e alta rusticidade (COSTA,1997). Essas pesquisas desenvolvidas pelos institutos de pesquisa foram responsáveis pelo aumento considerável nos mais importantes indicadores tecnológicos da avicultura de corte. A Tabela 8 informa sobre o avanço da conversão alimentar e da idade de abate. 
Tabela 8. Evolução da avicultura de corte no Brasil

\begin{tabular}{cccc}
\hline Ano & Peso Vivo (kg) & Conversão Alimentar & Idade de Abate \\
\hline $\mathbf{1 9 3 0}$ & 1.500 & 3.50 & 15 semanas \\
$\mathbf{1 9 4 0}$ & 1.550 & 3.00 & 14 semanas \\
$\mathbf{1 9 5 0}$ & 1.580 & 2.50 & 10 semanas \\
$\mathbf{1 9 6 0}$ & 1.600 & 2.25 & 8 semanas \\
$\mathbf{1 9 7 0}$ & 1.700 & 2.15 & 7 semanas \\
$\mathbf{1 9 8 0}$ & 1.800 & 2.05 & 8 semanas \\
$\mathbf{1 9 8 4}$ & 1.860 & 2.00 & 47 dias \\
$\mathbf{1 9 8 8}$ & 1.940 & 2.00 & 47 dias \\
$\mathbf{1 9 9 4}$ & 2.050 & 1.98 & 45 dias \\
$\mathbf{1 9 9 8}$ & 2.150 & 1.95 & 45 dias \\
$\mathbf{2 0 0 0}$ & 2.250 & 1.88 & 43 dias \\
$\mathbf{2 0 0 4}$ & 2.390 & 1.83 & 43 dias \\
$\mathbf{2 0 0 9}$ & 2.440 & 1.76 & 41 dias \\
$\mathbf{2 0 1 1 *}$ & 2.730 & 1.74 & 41 dias \\
\hline
\end{tabular}

Fonte: Relatório Anual da União Brasileira de Avicultura. UBA (2009)

*Obtido das Tabelas Brasileiras para Aves e Suínos de 2011.

Outro resultado importante da pesquisa para a avicultura de corte se refere ao número de aves alojadas por $\mathrm{m}^{2}$. Em 1970, eram alojadas menos de 10 aves por $\mathrm{m}^{2}$, enquanto na década de 1990, nas criações intensivas, são acomodadas 20 aves por $\mathrm{m}^{2}$.

\subsection{Bovino de corte}

No começo do século XX, o meio científico brasileiro iniciou seus esforços no sentido de dotar o país dos meios necessários ao avanço da genética bovina. A pavimentação das atividades ficou a cargo de iniciativas governamentais, particularmente, pela estruturação de estações

7 As informações sobre bovinocultura de corte, cujas fontes não são indicadas no texto, foram gentilmente fornecidas pelo Professor Mário Fonseca Paulino, do Departamento de Zootecnia da Universidade Federal de Viçosa. 
experimentais destinadas à atividade de seleção. Após os trabalhos iniciais de avaliação e caracterização biológica, foram iniciados, na década de 1940, trabalhos que tinham como orientação o desenvolvimento de uma raça nacional, que aliasse o maior potencial de produção, verificado no gado europeu, à rusticidade e à adaptabilidade das raças importadas da Índia. No início dos anos de 1950, foi iniciado, na Estação Experimental de São José do Rio Preto, SP, o trabalho de seleção da raça Nelore. É também dessa época a criação, em Ribeirão Preto, SP, de uma estação experimental voltada para seleção e criação da raça Gir. Ao final desta mesma década, foi criada, em Uberaba, MG, a Fazenda Experimental de Criação. Essas duas estações foram pioneiras no estudo de melhoramento genético do gado zebu no Brasil (FILHO, 2009).

A pecuária de corte no Brasil se sustenta no tripé gado zebuíno, capim braquiária e suplemento múltiplo, tendo sido esses três elementos objeto de grande esforço de pesquisa nas universidades e centros de pesquisa. $\mathrm{O}$ gado zebuíno foi submetido a intenso processo de melhoramento genético, o que tem permitido que animais tratados com pastagem e suplemento múltiplo sejam abatidos, hoje, em propriedades comerciais, com 16 arrobas, aos 20 meses de idade. Na década de setenta, um animal com esse mesmo peso demorava entre 36 e 48 meses, o dobro do tempo, para ir ao abate. Atualmente, são abatidos no Brasil 41,2 milhões de animais por ano, sendo aproximadamente $7 \%$ desses animais provenientes de confinamentos (ANUALPEC 2012). Na década de 1970, eram abatidos apenas 20,0 milhões de animais. Foi importante para a melhoria genética dos rebanhos, em condição de campo, o desenvolvimento de indicadores do mérito dos reprodutores para a características desejadas e da técnica de inseminação artificial de tempo fixo.

A introdução no país dos capins do gênero braquiária, de origem africana, na década de 1970, contribuiu muito para reduzir a sazonalidade no ganho de peso dos rebanhos. Os capins braquiária ainda permitiram a incorporação do Cerrado às áreas de pastagem nacional. Atualmente, 54,0 milhões de hectares do cerrado são pastagens de alta qualidade (EMBRAPA). 
$\mathrm{Na}$ área de nutrição animal, o esforço de pesquisa da Empresa de Pesquisa Agropecuária de Minas Gerais (Epamig) e das universidades foi fundamental para o desenvolvimento do Suplemento Múltiplo na década de 1980. Esse suplemento complementa os nutrientes inexistentes na pastagem, permitindo o ganho de peso continuado do rebanho e uma taxa de abate que aumentou de $16,0 \%$ na década de 1970 para a atual de $22,2 \%{ }^{8}$ (ANUALPEC, 2012). A medicina veterinária preventiva, combatendo a febre aftosa e a brucelose, contribui grandemente para a qualidade da carne produzida.

\section{Conclusão}

Uma importante contribuição das universidades para o desenvolvimento do agronegócio foi a formação de capital humano, isto é, de profissionais de elevada qualidade técnica para suprir a demanda do sistema de pesquisa, de extensão, de ensino das ciências agrárias e dos elos das cadeias do agronegócio. As universidades cumpriram esse papel apoiadas nas bases sólidas em que foram instituídas, na competência e idealismo dos seus professores, nas agências financiadoras Capes e CNPq e nos programas firmados com universidades estrangeiras.

A contribuição das universidades na pesquisa e desenvolvimento tecnológico agrícola foi e continua sendo de grande relevância nas áreas de melhoramento genético de plantas e animais, controle de pragas e doenças, correção e fertilidade dos solos, sequenciamento genômico, agricultura de precisão, desenvolvimento de organismos geneticamente modificados e desenvolvimento de produtos.

Alguns dos produtos mais beneficiados com a pesquisa foram: milho, soja, café, cana-de-açúcar, eucalipto, bovino de corte e frango.

8 A taxa de abate refere-se ao número de animais abatidos em relação ao rebanho total. 


\section{Referências}

ANUALPEC 2012. 2012. Informa Economics FNP, Prol Editora Gráfica, São Paulo, SP.

BARRETT, C.B., M.R. CARTER; C. P. TIMMER. 2010. "A CenturyLong Perspective on Agricultural Development," American Journal of Agricultural Economics 92: 447-468.

BORGES, J. M.; SABIONI, G. S. MAGALHÃES, G.F.P., 2006. A Universidade Federal de Viçosa no Século XX. Editora UFV. Segunda edição.

CAFEICULTURA. Produtividade do café em Minas Gerais cresce mais que o dobro. Disponível em: http://www.revistacafeicultura.com.br

CAPES - Coordenação de Aperfeiçoamento de Pessoal de Nível Superior. Número de Cursos de Pós-Graduação. Disponível em: http://www. capes.gov.br/

CEPEA - Centro de Estudos Avançados em Economia Aplicada - ESALQUSP. PIB do Agronegócio. Access: http://cepea.esalq.usp.br/pib/

CIB - Conselho de Informações sobre Biotecnologia., 2008. Eucalipto: oportunidades para um desenvolvimento sustentável. Editora CIB. 20p.

CNPq - Conselho Nacional de Desenvolvimento Científico e Tecnológico. Número de Bolsa de Mestrado e Doutorado. Access: http://www.cnpq.br/

COELHO, F.M.G., 1992. A produção científico-tecnológica para a agropecuária: da ESAV à UREMG, conteúdos e significados. Universidade Federal de Viçosa. Dissertação de Mestrado (Extensão Rural). 243p. 
COSTA, A.J.D., 1997. Agroindústria brasileira contemporânea: inovações organizacionais e transformações tecnológicas na avicultura. Université da La Sorbonne Nouvelle Paris III. Tese de Doutorado. 352p.

EMBRAPA. Centro Nacional de Pesquisa Agropecuária do Cerrado (CPAC). Access: http://www.cpac.embrapa.br/

EUCLIDES FILHO, K., 2009. Evolução do melhoramento de bovinos de corte no Brasil. Revista Ceres, Viçosa, 56 (5): 620-626.

FALEIRO, F.G.; SOUSA, E.S., 2007. Pesquisa, desenvolvimento e inovação para o cerrado. Embrapa Cerrados, Planaltina, DF. 133 p.

FERNANDEZ, A., 1991. The Purdue-Viçosa Project: a case study in institution building. Purdue Universiy. Thesis of Doctor of Philosophy. 202p.

FERRARI, A. F., 2001. José Pelúcio Ferreira e a pós-graduação no Brasil. Capes, Biblioteca Anísio Teixeira, Série Biografias, Paralelo 15. $195 p$.

FERREIRA, M. M., 1993. Retorno aos investimentos em pesquisa e assistência técnica na cultura do café em Minas Gerais. Universidade Federal de Viçosa. Dissertação de Mestrado (Economia Rural). 157p.

GASQUES, J.G.; BASTOS, E.T.; VALDES, C.; BACCHI, M.R., 2012. Produtividade da Agricultura Brasileira e os Efeitos de Algumas Políticas. Revista de Política Agrícola, 23 (3): 83-92, July, August and September.

MAPA - Ministério da Agricultura e do Abastecimento -. Secretaria de PolíticaAgrícola. Access: http://www.agricultura.gov.br/

MENDES, G. M.; TEIXEIRA, E.C.; SALVATO, M.A., 2012. Produtividade Total dos Fatores e Crescimento Econômico na 
Agropecuária Brasileira: 1970-2006. In: Anais do 50. Congresso da SOBER, Vitória, ES, 22 a 25 de julho.

NOVAIS, R.F.; ALVAREZ, V.H.; BARROS, N.F.; FONTES, R.L F.; CANTARUTTI, R.B.; NEVES, J.C.L., 2007. Fertilidade do Solo. Editora UFV.

SCHULTZ, T. W. (1964). Transforming traditional agriculture. New Haven: Yale University Press.

SCHULTZ, T. W. (1971). Investment in Human Capital: The Role of Education and of Research. New York: Free Press.

SEDIYAMA, C.S; CARNEIRO, J. E. S.; FRITSCHE-NETO, R.; SEDIYAMA, T.; BARBOSA, M. H. P.; GALVÃO, J.C.C.; SOUZA, M.A., 2012. Contribution of the universities to the development of field crop cultivars. Crop Breeding and Applied Biotechnology S2: 121-130.

UBA - União Brasileira de Avicultura., 2009. Relatório anual. 
REVISTA DE ECONOMIA E AGRONEGÓCIO, VOL.11, $N^{\circ} 1$ 